\title{
EL IMPACTO DE LA CIENCIA Y TECNOLOGÍA EN LA CULTURA DEL TURISMO COMUNITARIO: EL CASO SALASACA - ECUADOR
}

\author{
IMPACT OF SCIENCE AND TECHNOLOGY IN THE CULTURE OF \\ COMMUNITY TOURISM: THE SALASACA CASE - ECUADOR
}

\author{
Esther Mullo Romero' \\ Efrén Mendoza Tarabó ${ }^{2}$ \\ Jhony Yumisaca Tuquinga ${ }^{2}$ \\ 1 Universidad de la Habana, Cuba. emulloromero@gmail.com \\ 2 Universidad Estatal Península de Santa Elena, Ecuador.
}

\section{RESUMEN}

El creciente impulso de la Ciencia y la Tecnología, ha logrado tener acercamientos con lugares que jamás se ha imaginado el hombre y se la ve como una técnica de vital importancia para el sector del turismo. En este artículo se propone como táctica metodológica la focalización analítica de la ciencia y tecnología en la cultura del turismo comunitario de Salasaca en la provincia de Tungurahua. El objetivo que se persiguió en este trabajo está ligado al impacto de la ciencia y tecnología en la cultura del turismo comunitario. Como conclusión se plantea el cambio en la manera de promocionar un lugar, no solo por lo natural sino por lo científico y tecnológico que puede aportar al visitante. De aquí se derivan una serie de indicadores cualitativos que sirven para encarar, desde el punto de vista teórico, la comprensión general de la ciencia y la tecnología en la cultura del turismo comunitario y así mismo, son útiles para la evaluación de la sostenibilidad de proyectos y experiencias del turismo en las comunidades.

Palabras clave: Ciencia, Tecnología, Cultura, Turismo, Comunidad

\begin{abstract}
The growing momentum of science and technology, has achieved approaches with places that man has ever imagined and sees it as a technique of vital importance for the tourism sector. In this article the analytical focus of science and technology in the culture of the Community tourism, where they worked with the Salasaca community in an in-depth study is proposed as methodological tactic. The objective, which is pursued in this work, is linked to the impact of science and technology in the culture of Community tourism. As conclusion is raises, the change in the way of promoting a place, not only by it natural but also by it scientific and technology that can contribute to the visitor. From this, a series of qualitative indicators are derived that serve to approach, from the theoretical point of view, the general understanding of science and technology in the culture of community tourism and also are useful for the evaluation of the sustainability of projects and experiences of Tourism in Communities.
\end{abstract}

Keywords: Science, Technology, Culture, Tourism, Community

Recibido: 10/10/2017; $\quad$ Aprobado: 07/12/2017; $\quad$ Publicado: 19/12/2017

\section{1.- INTRODUCCIÓN}

Las presiones de las nuevas corrientes turísticas mundiales gestadas en las dos últimas décadas se han traducido en un creciente número de proyectos de turismo en comunidades indígenas o rurales, mediante el impulso de agentes externos como ONGs, empresas privadas, instituciones públicas y de cooperación internacional.

De esta manera en las últimas décadas varios países de América Latina han sido fuertemente sacudidos por las agrupaciones geográficas de los movimientos sociales indígenas. Varios Estados han reaccionado aprobando nuevas leyes y constituciones que 
reconocen la diversidad de culturas, así como los derechos colectivos de dichas poblaciones. Este ha sido el caso, de Guatemala, Nicaragua, Colombia, Ecuador, Perú, Bolivia, Chile y Brasil.

De este modo el turismo comunitario ha trascendido las bases del turismo natural, creando un entorno amigable entre hombre y naturaleza y en cualquier otro ámbito del sector turístico (turismo cultural, vivencial, de aventura...).

Delgado ${ }^{1}$ afirma "En su devenir histórico, el hombre ha logrado cambiar sustancialmente los modos de vivir y ha superado dependencias ancestrales con respecto a la Naturaleza". De ahí la importancia para que organizaciones y comunidades indígenas vean al turismo como medio de reivindicación y autogestión sobre sus territorios y recursos, que sirve para reclamar un lugar en el Estado y el mercado, a manera de táctica turística.

El desarrollo científico y tecnológico es uno de los factores más influyentes sobre la humanidad contemporánea y la globalización mundial actual. "La ciencia se presenta como una institución social, como un sistema de organizaciones científicas, cuya estructura y desarrollo se encuentran estrechamente vinculados con la economía, la política y los fenómenos culturales" 1.

Dado el carácter universal de la ciencia y su influencia se extiende a todos los campos de la sociedad. Para Núñez ${ }^{2}$ "La ciencia y la tecnología son, ante todo, procesos sociales... Lo que convierte al conocimiento en un recurso significativo que lo promueve y desarrolla".

En todos los casos es deseable que los logros científicos conseguidos lleguen a la sociedad. La importancia de la ciencia y la tecnología en la gestión turística es un factor primordial sobre todo para el desarrollo económico y social, por tal razón es menester contar con individuos bien formados que protagonicen los desafíos de la innovación tecnocientífica, por otra parte no será posible afrontar con éxito los retos de la industrialización turística, que tributen en beneficio de la todos.

La consideración de la ciencia, la tecnología y la sociedad como elementos en continua interacción facilita la aproximación en la gestión turística, favorece también el desarrollo de actitudes de interés hacia esos temas y crea las condiciones para la aparición de inquietudes para profundizar en ellos que serán la base de vocaciones científicas para el futuro.

\section{2.-OBJETIVOS}

Identificar el impacto de la ciencia y tecnología en la cultura del turismo comunitario de Salasaca, Ecuador.

Analizar la importancia del desarrollo de la ciencia y tecnología en la cultura del turismo comunitario realizado en la comunidad de Salasaca.

\section{3.-FUNDAMENTACIÓN}

\subsection{La ciencia y tecnología}

La globalización ha permitido acceder a distintos productos y servicios de varios lugares del mundo, no podría ser de otra manera los relacionados con el sector turístico, a su vez se encuentran fortalecidos por la tecnología y la ciencia, que permiten acceder a un mayor número de posibilidades, que están acordes a la sociedad de la información y conocimiento que estamos viviendo. No sorprende, entonces, que la ciencia y la tecnología jueguen un papel importante en la práctica de la actividad turística y en la mejora de la eficiencia de las organizaciones y la entrega del servicio. La ciencia y la tecnología han sido catalizadores importantes de los cambios experimentados de la industria en los últimos 30 años.

En esas condiciones está claro que la tecnología vuelve a ocupar un lugar central de debate en la sociedad moderna, como hace tres siglos ocurrió con la máquina de vapor y la revolución industrial.

Al mismo tiempo, la red de información permite a las empresas alcanzar ventajas importantes en el mercado, logrando satisfacer las necesidades de todos, adquiriendo vínculos de confianza y garantía en cada producto ofertado ya que facilita la elección acertada del destino y genera mayor seguridad a la hora de viajar.

\subsection{Ciencia, Tecnología y Sociedad en el Turismo}

Ciencia, Tecnología y Sociedad (CTS) es más que una relación de esos tres 
conceptos, hoy se acentúa la importancia de esenciales interacciones entre ellos. En las últimas décadas esa vinculación entre ciencia, tecnología y sociedad ha sido más intensa y ha comenzado a constituir un tema de reflexión, pues la ciencia y la tecnología condicionan las formas de vida humana en el planeta, generando cambios en muchas áreas así como en el turismo².

El desarrollo científico y tecnológico es una de los factores más influyentes sobre la sociedad contemporánea. La globalización mundial, polarizadora de la riqueza y el poder, sería impensable sin el avance de las fuerzas productivas que la ciencia y la tecnología que han hecho posibles.

"Hoy en día los estudios CTS constituyen una importante área de trabajo en la investigación académica, política y educación. En este campo se trata de entender los aspectos sociales del fenómeno científico y tecnológico, tanto en lo que respecta a sus condicionantes sociales como en lo que atañe a sus consecuencias sociales y ambientales. Su enfoque general es de carácter crítico respecto a las visiones clásicas de ciencia y tecnología donde sus dimensiones sociales son ocultadas, donde concurren disciplinas como la Filosofía, la Historia, la Sociología de la Ciencia y la Tecnología, entre otras. La educación en CTS persigue precisamente cultivar ese sentido de responsabilidad social de los sectores vinculados al desarrollo científico tecnológico y la innovación" 2 .

El impulso a los estudios de Ciencia y Tecnología debe entenderse como una respuesta a los desafíos sociales e intelectuales que se han hecho evidentes en décadas pasadas así como en el presente. Su estudio ha sido definido como $^{3}$ : "Exponer una interpretación de la ciencia y la tecnología como procesos sociales, es decir, como complejas empresas en las que los valores culturales, políticos y económicos ayudan a configurar el proceso que, a su vez, incide sobre dichos valores y sobre la sociedad que los mantiene".

Es por ello que la ciencia y la tecnología ha transformado la manera en que se realiza turismo, ya que esta es una de las industrias más grandes a nivel mundial. No sorprende, entonces, que jueguen un papel importante en la práctica de la actividad turística y en la mejora de la eficiencia de las organizaciones y la entrega del servicio.
Ante esta situación, todos los involucrados necesitan orientar y centrar su modelo de gestión de negocio en las necesidades de sus clientes para poder afrontar los cambios y los retos estratégicos que tienen planteados dentro de esta área que es el turismo.

Dado que las organizaciones se encuentran inmersas en un ambiente de alta productividad y competitividad internacional, necesitan de constantes desarrollos científicos y tecnológicos que le conduzcan a la innovación de productos, servicios y tecnologías, entre otros, que representen la oportunidad de negocios altamente rentables, además del beneficio para la comunidad académica y científica de la sociedad en general.

En principio la función de la ciencia se vincula a la adquisición de conocimientos, al proceso de conocer, cuyo ideal más tradicional es la verdad, en particular la teoría científica verdadera. La objetividad y el rigor son atributos de ese conocimiento. Agazzi ${ }^{4}$ admite que en su evolución la "ciencia ha cambiado considerablemente, desde una ciencia basada en la contemplación, para luego orientarse al descubrimiento y finalmente, lo cual sería su rasgo contemporáneo, a la investigación"; Kröber ${ }^{5}$ resume el tema así: "entendemos la ciencia no sólo como un sistema de conceptos, proposiciones, teorías, hipótesis, etc., sino también, simultáneamente, como una forma específica de la actividad social dirigida a la producción, distribución y aplicación de los conocimientos acerca de las leyes objetivas de la naturaleza y la sociedad. Aún más, la ciencia se nos presenta como una institución social, como un sistema de organizaciones científicas, cuya estructura y desarrollo se encuentran estrechamente vinculados con la economía, la política, los fenómenos culturales, con las necesidades y las posibilidades de la sociedad dada".

De esta manera se le puede analizar como sistema de conocimientos que modifica nuestra visión del mundo real y enriquece nuestro imaginario y nuestra cultura; se le puede comprender como proceso de investigación que permite obtener nuevos conocimientos, los que a su vez ofrecen posibilidades nuevas de manipulación de los fenómenos; es posible atender sus impactos prácticos y productivos, caracterizándola como fuerza productiva que propicia la transformación del mundo y es fuente de riqueza; la ciencia también se 
nos presenta como una profesión debidamente institucionalizada portadora de su propia cultura y con funciones sociales bien identificadas.

La razón por la cual es posible apreciar tantas facetas diferentes de la ciencia es porque ella constituye un fenómeno complejo cuyas expresiones históricas han variado considerablemente. Por eso las definiciones de ciencia resultan escurridizas y a veces inalcanzables.

La relación que existe entre ciencia y tecnología, es que ambas necesitan de un método experimental para ser confirmadas, puede ser demostrable por medio de la repetición. Por otra parte, la ciencia se interesa más por el desarrollo de leyes, las cuales son aplicadas por la tecnología para sus avances.

Existe una tecnología para cada ciencia, es decir, cada rama posee un sistema de tecnología diferente, que permite un mejor desarrollo para cada una de ellas.

Cabe recordar, que la tecnología se percibe con los sentidos, es decir, podemos observarla y verla, en un mundo que depende de forma creciente de la ciencia y la tecnología. Los procesos de producción, las fuentes de alimentación, la medicina, la educación, la comunicación o el transporte son todos campos cuyo presente y futuro están fuertemente ligados al desarrollo tecnológico y científico.

La ciencia y la tecnología han contribuido a mejorar nuestras condiciones de vida, aumentando su calidad y transformando nuestro entorno. En la civilización tecnológica que vivimos, es una red que abarca los más diversos sectores de la actividad humana "un modo de vivir, de comunicarse, de pensar, un conjunto de condiciones por las cuales el hombre es dominado ampliamente, mucho más que tenerlos a su disposición" 6 .

Price $^{7}$ define a la tecnología "como aquella investigación cuyo producto principal es, no un artículo, sino una máquina, un medicamento, un producto o un proceso de algún tipo".

Mientras que Quintanilla ${ }^{8}$ también la define como: "técnicas industriales de base científica. Para estas reservamos el término tecnología". (.....) Y también: "Las tecnologías son complejos técnicos promovidos por las necesidades de organización de la producción industrial, que promueven a su vez nuevos desarrollos de la ciencia" (...).

La influencia de las tecnologías en la vida social es, hoy más que nunca, de gran intensidad y el sector turístico está bajo la influencia de las nuevas tecnologías (NT). La Comisión Económica para América Latina y el Caribe $(C E P A L)^{9}$ creó una herramienta que calcula el Índice de Especialización Tecnológica (IET), la cual ha sido utilizada en la industria como medida del porcentaje de nuevas tecnologías aplicadas al turismo, tomando como referencia las nuevas tecnologías desarrolladas.

Por lo arraigada que está la tecnología en la práctica turística, se podría afirmar que toda forma de turismo, hasta cierto punto y en mayor o menor grado, es tecnológica, por la participación que ha tenido la tecnología en su consecución. Sin embargo esto, más que como un resultado, único e inexorable, debe ser vista como un proceso social, una práctica, que integra factores psicológicos, sociales, económicos, políticos, culturales; siempre influido por valores e intereses.

La ciencia contemporánea, según se presenta, se orienta cada vez más a objetos prácticos, a fomentar el desarrollo tecnológico y con este la innovación, a su vez, que la tecnología, es cada vez más dependiente de la actividad y el conocimiento científico, lo que sugiere y apunta que los clásicos límites atribuidos a ciencia y tecnología se están volviendo borrosos y aún más, disolviéndose frente a un complejo ciencia - tecnología. "El guión que une los términos de 'ciencia tecnología' indica esa unión esencial. La nueva ciencia es, por su esencia, tecnológica" 10.

El término tecno-ciencia que designa el complejo entramado de la ciencia y la tecnología contemporáneas tiene una carga conceptual especial. No sólo indica que con el paso de la ciencia académica a la ciencia gubernamental e industrial, sobre todo en el siglo XX, ciencia y tecnología han llegado a ser prácticamente inseparables en la realidad. También señala una nueva imagen de la ciencia y la tecnología que los actuales estudios han ido destacando frente a las concepciones tradicionales. Una de las ideas características es que la ciencia no se puede reducir a los científicos ni la tecnología a los tecnólogos, sino que 
ambas forman parten de complejas redes junto con otros agentes y entornos simbólicos, materiales, sociales, económicos, políticos y ambientales.

Se trata de tomar conciencia de la naturaleza de la actividad científica y tecnológica contemporánea. La Biotecnología, la Farmacología, la Química Sintética serían algunos ejemplos, entre muchos, que ilustran la naturaleza de la tecno-ciencia. "Los términos 'tecno-ciencia' y 'tecno-científico' señalan, a la vez, el entrelazamiento entre los dos polos y la preponderancia del polo técnico y, además, son apropiados para designar la actividad científica contemporánea en su complejidad y originalidad".

Las complejas interacciones entre ciencia, tecnología, sociedad y naturaleza forman una unidad de hecho inseparable y un tupido entramado que sólo pueden abordarse en el marco de estudios globales. El presente trabajo parte de la comprensión y el análisis que integra todas esas dimensiones de la ciencia como coordenadas de la misma en un espacio tecno-cultural global. Se podría pensar que el actual entramado de ciencia, tecnología, sociedad y naturaleza que constituye el núcleo de lo que se llama tecno-ciencia es exclusivo de nuestra época, pero lo cierto es que ha existido con diferentes formas a lo largo de las tradiciones científicas, junto con las decisivas transformaciones económicas, sociales, culturales y ambientales que estas están operando a escala mundial.

Pues bien, la tecno-ciencia se ha convertido en un recurso estratégico político y económico tanto para los Estados como para las industrias, no se debe desconocer que el desarrollo de la misma puede aportar ventajas al bienestar de la sociedad, sobre todo si son de comunidades que quieren ser protagonistas de su propio desarrollo en todos los ámbitos, sin dejar de lado la conservación del medio ambiente, además, el desarrollo aporta nuevas formas de relación y nuevos valores. . En una nota publicada en el diario "Clarín" Filmus"1 afirma: "Una educación que forme ciudadanos participativos y solidarios, que utilicen críticamente las tecnología-ciencia, ayudará a la construcción de una sociedad más justa, humana y sin exclusiones".

Esta perspectiva implica una transformación de los procesos de oferta y demanda inmersos, es decir, en el caso de actividades productivas, como es el caso del Turismo, en donde además se genera una diversificación de servicios, viene a ser parte de la interconectividad con el servicio y la calidad, en la sociedad del conocimiento de hoy, ello significa que la ciudadanía debe incluir la capacitación y la instrucción dentro de los principios científicos básicos.

Con el pasar de los años el desarrollo de la tecno-ciencia ha dado un impulso a la prestación de servicios turísticos y también a la conservación de las áreas naturales que poseen una riqueza única de flora y fauna, los beneficios que se pretenden alcanzar con el impacto de la tecno-ciencia sería lograr el desplazamiento de personas a lugares cuya civilización muestra avances importantes en conocimientos y aplicaciones técnicas y científicas. Hay dos variantes en la práctica del turismo con la tecno-ciencia: una es aquella en que el turista se desplaza a países en donde se producen los avances (para verlos antes de que lleguen a sus países de origen) y la otra, es aquella donde los turistas se desplazan a lugares que, si bien producen avances, más se conocen por promoverlos a través de su especificidad.

Es por ello, que el turismo comunitario se puede convertir en un campo de desarrollo estratégico para impulsar la economía, social, ambiental y cultural, que en la actualidad exige la demanda del mercado, así como la búsqueda de actividades económicas compatibles con el cuidado, conservación y mantenimiento del medio ambiente. Por tanto, los beneficios derivados del desarrollo turístico comunitario enmarcan mejoras en la calidad de vida de todos quienes interactúan, combinando aprendizaje, buen servicio en la calidad del ambiente y la cultura local intacta.

Una de las razones principales que le permite al turismo apoyarse en la informática, es el hecho que la actividad turística es interterritorial, que puede promocionar y comercializar sus servicios sin importar la distancia en la que se encuentre el potencial cliente, éstas oportunidades las pueden aprovechar hoteles, restaurantes, agencias de viajes y operadoras, permitiéndoles obtener ventajas competitivas como de rapidez en comunicaciones, reducción de costos y errores, información actualizada en tiempo real que tratarán de satisfacer las 
necesidades primordiales de los turistas como son alojamiento, alimentación y descanso.

\section{Impacto de la ciencia y tecnología en la cultura del turismo comunitario}

La ciencia y la tecnología, como parte del ambiente humano, está siempre ligada a la cultura. La presencia de éstas genera cambios en hábitos y costumbres de las relaciones individuo-sociedad, y en todos los aspectos:

Las nuevas tecnologías, tanto analógicas como digitales, aportan al arte una nueva sensibilidad a la hora de representar y pensar las imágenes y otros productos artísticos. La influencia se ve reflejada en las nuevas formas de fotografía, videos, pinturas entre otros.

Una serie de desarrollos tecnológicos cambiaron y siguen cambiando no sólo la forma cómo se escucha música, sino también, cómo se usa y cómo se relacionan las personas a través de ella. Se modificó también la forma en que los músicos crean, distribuyen sus trabajos e interactúan con el público.

De esta manera la presencia de las nuevas tecnologías también en nuestra sociedad está generando innumerables cambios en hábitos y costumbres de las relaciones individuo-sociedad, esto es, en todo aspecto de la actividad humana.

Una de las costumbres que han cambiado a causa de los avances tecnológicos son:

Ver TV en familia, compartir en familia

\section{Escribir cartas}

\section{Ir a la biblioteca a realizar tareas}

El entretenimiento online ha logrado alejar a los niños, y en general a todas las generaciones, de los parques, los museos, los centros comerciales...

Es por ello que la búsqueda de valoración en torno a la Cultura de nuestros pueblos se encuentra delineada en la política cultural de la UNESCO ${ }^{12}$, que la reconoce como un factor esencial del desarrollo. Así en la Conferencia ASICULT en, Yogyakarta ${ }^{13}$ se preconizó:

"...La búsqueda de modelos originales de desarrollo, que pudieran garantizar un equilibrio entre los valores materiales y espirituales, sociales e individuales, entre la preservación de las culturas nacionales y la asimilación necesaria de la ciencia y la tecnología".

$Y$ se puntualiza además, 'no debe confundirse mantenimiento de la tradición con rechazo del progreso científico tecnológico", es decir, es en nuestras regiones donde la evaluación de la tecnología y las políticas que de ella se derivan, alcanzan un papel primordial, ya que es necesario que la práctica tecnológica y científica se controle por los países usuarios, y se aplique de acuerdo a modelos adaptados al contexto de estas regiones $y$ en consonancia con las necesidades reales de su estrategia de desarrollo. Estos elementos están en la base de lo que hoy implica el concepto de "Desarrollo Sustentable".

Es así que se puede evidenciar que no todos los estados controlan el uso y poder que la tecnología y la ciencia puede dar a ciertos sectores, aplicando de una manera descontrolada y perdiendo los límites de tolerancia, tanto ecológicos como sociales, eliminando las capacidades autoreguladoras de nuestras sociedades.

Las tendencias de las tecnologías son precisamente la: aceleración de los cambios tecnológicos y la globalización lo que implican un impacto a escala global de la misma, entendido en el sentido que no hay región del planeta que no perciba su influencia (por exceso o por defecto).

Como ya se habló anteriormente la ciencia y la tecnología generan cambios que aun pueblos, ciudades y estados no han podido controlar ocasionando pérdidas socioculturales, pero poco o nada se sabe del impacto en el turismo comunitario en Ecuador, en donde la ciencia y la tecnología se ha visto empleada para el turismo en muchas comunidades del país, tanto así, es que el turismo comunitario es en los actuales momentos es conocida como una "marca internacional" y un elemento de creciente interés en la oferta y demanda turística de los países de Latino América, ya existen diversidad de experiencias de turismo comunitario en Asia, en África, Canadá, Australia, Estados Unidos y Nueva Zelanda, en los países de habla hispana, desde México hasta Bolivia, pasando por Centroamérica y los países andinos, e incluso Argentina, encontramos tanto experiencias locales como Federaciones Nacionales y redes que agrupan dichas iniciativas y trazan su propio camino en el mundo del turismo. 
Ecuador como el país precursor, por el peso cuantitativo y cualitativo de sus experiencias de turismo comunitario, desde los años ochenta, ha logrado convertirlo en una actividad estratégica para muchas comunidades ya que actúa como catalizador de diferentes procesos.

De esta forma, el turismo comunitario se convierte en un campo estratégico de desarrollo social, económico y cultural, que parece encarar con éxito varias de las más acuciantes expectativas del mundo actual.

Así para sus actores "Turismo comunitario es toda actividad turística solidaria que permite la participación activa de la comunidad desde una perspectiva intercultural, manejo adecuado del patrimonio natural y valoración del patrimonio cultural, basados en un principio de equidad en la distribución de los beneficios locales"14.

El impacto de la ciencia y la tecnología ha contribuido al crecimiento masivo del turismo y al aumento del volumen de la oferta y la demanda. Esto convierte a las Tecnologías de en uno de los elementos clave de la industria turística, ya que son el instrumento para la comercialización, la distribución y las funciones de ajuste de las comunidades turísticas a la vez que a los consumidores les permite optimizar el valor de su dinero y tiempo cuando viajan y hacen turismo, siendo así la comunidad quien domina la tecnología y no viceversa

\section{4.- METODOLOGÍA}

Se ha partido de la afirmación que la ciencia y la tecnología no es un factor de conflicto sino una herramienta que aporta gran valor al desarrollo del turismo comunitario.

Para demostrar si esta premisa es real, se realizó un estudio empírico, descriptivo y exploratorio de manera que sea posible aportar algo a quienes están interesados en este tema.

Como fuente primaria se aplicó un cuestionario con 10 preguntas, dirigido a Directores de Investigación y dos investigadores por cada una de las siguientes universidades: Universidad Técnica de Ambato, Uniandes, Universidad Tecnológica Indoamérica, Universidad Católica del Ecuador Sede Ambato.
Con este cuestionario, se buscó medir las dimensiones científicas y tecnológicas en la cultura del turismo comunitario.

\section{5.- RESULTADOS}

\section{Importancia del Desarrollo de la Ciencia y Tecnología en la Cultura del Turismo Comunitario: El Caso de la Comunidad de Salasaca}

La importancia del desarrollo de la ciencia y la tecnología en la cultura del turismo comunitario, actualmente, ha logrado tener acercamientos con lugares que jamás se ha imaginado el hombre, motivado por la necesidad de conocer otros lugares ya sea por diversión, trabajo o recreación familiar, generando una buena economía en los sectores de mayor afluencia turística, todo gracias a la contemplación teórica y práctica que hoy en día se la ve como una técnica de vital importancia para el sector del turismo.

Por lo tanto el turismo y su aplicación científica, se encuentra en un momento excepcional, que urge la definición de nuevos desafíos y herramientas en la planificación, desarrollo y manejo de los destinos turísticos, especialmente a escala local. Por lo que, la idea del "pensar en lo global para actuar en lo local" es un concepto filosófico que parte desde lo local manejando criterios globales, que es aceptado en la administración de este milenio. Existen, hablando en positivo, nuevas posibilidades y enormes potenciales desde la actividad turística que pueden ayudar a la comunidad a configurarla de manera más humana, más equitativa y más competitiva, proponiendo como forma de combatir el cambio climático la utilización de energías y tecnologías alternativas.

En el turismo comunitario, dada la interrelación entre los distintos actores que lo componen hoteles, transportes, restaurantes, agencias de viajes, etc.-, los sistemas de información tienen una gran importancia, ya que son un instrumento para mejorar la competitividad, dado su gran valor estratégico. En este sentido, conviene destacar la utilidad de los sistemas de información intraorganizativos, que se encargan de:

La gestión de la información interna (por ejemplo, programas informáticos de contabilidad, de gestión de almacenes, de gestión del personal, de fiscalidad, base de datos de clientes, etc.). De los sistemas de información intraorganizativos, que pretenden 
la interconexión electrónica de diversas organizaciones independientes (por ejemplo, centrales globales de reservas);

De otros medios de comunicación con el entorno, como, por ejemplo, Internet, que se ha convertido en un nuevo canal alternativo de distribución y una forma de hacer publicidad en el sector turístico.

El desafío es claro pero complejo: hacer viable el negocio y las empresas turísticas dentro del respeto a la participación de la comunidad en las decisiones y la planificación del turismo. Ello con tres vértices de gestión: beneficios sociales y económicos para el municipio y sus habitantes, calidad y sostenibilidad ambiental y desarrollo turístico acorde con la autenticidad cultural y señas de identidad local.

\section{La Tecnología y la Ciencia para el uso del Turismo Comunitario en la Comunidad de Salasaca.}

Vivimos en un mundo que depende de forma creciente de la ciencia y la tecnología. Los procesos de producción, las fuentes de alimentación, la medicina, la educación, la comunicación o el transporte son todos campos cuyo presente y futuro están fuertemente ligados, al desarrollo científico y tecnológico. Han contribuido de formas asombrosas a mejorar nuestras condiciones de vida, aumentando tanto la esperanza de vida como su calidad, y transformando los modos de interacción humana.

Dentro de la prestación de servicios turísticos, es importante que todas las actividades que se las realicen sean bajo un mismo principios de Sostenible es por ello que las llamadas energías renovables, se caracterizan por la transformación y aprovechamiento que pueden ofrecer por períodos relativamente largos (cientos de años) sin que se consuman ni se agoten.

Actualmente, gracias a la ciencia y tecnología, las energías renovables son de vital importancia para el sector del turismo, reduciendo los impactos ambientales derivados del uso de combustibles fósiles.

Entre las alternativas que pueden ofrecer la ciencia y la tecnología para un mejor turismo en la comunidad de Salasaca son el tratamiento de agua residual que no es más que un conjunto de métodos y procesos utilizados para modificar las propiedades del agua, ya sea en sus propiedades físicas, químicas y/o biológicas, con la finalidad de que el agua quede libre de sustancias o microorganismos para el consumo de la misma comunidad.
El concepto básico de todos los sistemas solares de agua caliente es utilizar la energía del sol para calentar o para precalentar el agua, de tal modo que reduzca los requerimientos de gas 0 electricidad para producir el agua caliente.

Este tipo de desarrollos al aplicarlos en la comunidad de Salasaca permitirá identificar oportunidades dentro de la esfera turística y en la industria, una vez que aquellas actividades logren involucrarse en el diseño e instrumentación del turismo, en la comunidad facilitarán la participación de dicha comunidad para contribuir al crecimiento económico.

La participación de la comunidad en el desarrollo del turismo comunitario ayudará a la reducción de la pobreza y la generación de fuentes de empleo y así de esta manea ayudará a la toma de decisiones de la comunidad al mismo tiempo que actúa como un estímulo para la producción de productos locales, como la gastronomía, la elaboración de ponchos, entre otros.

Luego del análisis de la información recolectada a través de las encuestas se resalta los siguientes resultados. Existe bajo impacto de la ciencia y la tecnología en la cultura del turismo comunitario en Salasaca.; debido fundamentalmente a que la actividad turística en esa comunidad se lo lleva de manera empírica y tradicional sin fundamentarse en aspectos científicos y tecnológicos, resultados que brindan la oportunidad para realizar trabajos de investigación en todos los ámbitos del turismo

El desarrollo de la ciencia y tecnología en los aspectos turísticos conlleva a un mejoramiento permanente del accionar turístico, este es el criterio de la mayoría de los entrevistados para esta investigación, por lo tanto la ciencia y la tecnología deben ser un recurso primordial para alcanzar un mayor impacto socioeconómico de la comunidad de Salasaca mediante el aprovechamiento de los recurso turísticos que posee este sector.

Otros de los resultados presentados por los encuestados es el alto interés de participación en la ciencia y tecnología para la cultura del turismo comunitario en Salasaca, reto inevitable de asumir para la Tecnología, como forma de organización social y parte de un sociosistema que deberá aplicar estrategias que no eximan la participación activa y creadora de la comunidad, reiterando siempre que el factor de desarrollo tecnológico y de promoción cultural, no borre el legado de las sociedades autóctonas para convertirlas en meros espectadores. 


\section{6.- CONCLUSIONES}

- La Tecnología debe ser entendida como forma de organización social integrada en socio sistemas a su vez más amplios e interdependientes

- Un desarrollo tecnocientífico en la comunidad Salasaca se convertiría en un instrumento de dominio, transformación y mejoramiento de las necesidades humana básicas que sería de ejemplo para muchas otras comunidades que buscan un desarrollo amigable con el medio ambiente y la sociedad por eso una correcta aplicación y uso tecnocientífico en este tipo de turismo es clave para el avance y desarrollo que hoy en día el gobierno se plantea en sus objetivos con en el control de la naturaleza, una mejora en la calidad de vida. En el sumak kausay.

- Asumir la Tecnología, como forma de organización social y parte de un sociosistema debe mover a estrategias que no eximan de la participación activa y creadora de nuestras comunidades en tanto factor de desarrollo tecnológico y promoción cultural, no borre el legado cultural de nuestras sociedades autóctonas y, no permita a la comunicación convertirse en incomunicación y desinformación.

\section{BIBLIOGRAFÍA}

1. Delgado, C. (2005) "Hacia un nuevo saber" La Bioética en la revolución contemporánea del saber; Editorial Félix Varela, La Habana.

2. Nuñez, J. (1999) "La Ciencia y la Tecnología", Problemas Sociales de la Ciencia y la Tecnología. GESOCYT; Editorial Félix Varela, La Habana.

3. Cutcliffe, S.H. (1990). Ciencia, Tecnología y Sociedad: un campo disciplinar, en Medina y Sanmartín (eds.) Ciencia, Tecnología y Sociedad. Estudios interdisciplinares en la universidad, en la educación y en la gestión pública. (pp. 20-41). Anthropos, Barcelona.

4. Agazzi, E.(1996): El bien, el mal V la ciencia. Las dimensiones éticas de la empresa científico-tecnológica.

5. Kröber, M. (1986): "Acerca de las relaciones entre la historia y la teoría del desarrollo de las ciencias", Revista Cubana de Ciencias Sociales, enero - abril, año IV, № 10, La Habana.

6. Fourez, G. (2000). La construcción del conocimiento científico. Madrid.

7. Price, D.J.S. (1980): "Ciencia y tecnología: Distinciones e interrelaciones", Estudios sobre sociología de la ciencia (Barnes, B. editor), Editorial Alianza Universidad, Madrid.

8. Quintanilla, M.A. (1991): Tecnología: un ensayo filosófico, EUDEBA, Buenos Aires.

9. CEPAL (Comisión Económica para América Latina y el Caribe) (1998a). El pacto.fiscal. Fortalezas, debilidades y desafíos (LC/G.1997/Rev.1), Santiago de Chile. Publicación de las Naciones Unidas, N' de venta: S.98.II.G.5.Conte, M. C. Ciencia, tecnología, ingeniería e innovación para el desarrollo. 2009.

10. Hottois, G.(1991): El Paradigma Bioético, Anthropos, Barcelona.

11.Filmus D.(2000) "La escuela, garantía de futuro" Diario Clarín. Seccion Educación. Sociólogo especializado en educación.

12.UNESCO (1999): "Declaración universal sobre la diversidad cultural: Identidad, diversidad y pluralismo" Recomendación sobre la salvaguardia de la cultura tradicional y popular, 1989 .

13.UNESCO (1973): "Conferencia intergubernamental sobre las politicas culturales" Yogyakarta. Asia.

14.FEPTCE. (2007). "Manual de Calidad para la Gestión del Turismo Comunitario en el Ecuador". Ed. FEPTCE. Quito. 